June 2001

UT-943

hep-th/0106027

\title{
Identity Projector and D-brane in String Field Theory
}

\author{
Yutaka MATSUO用 \\ Department of Physics, University of Tokyo \\ Hongo 7-3-1, Bunkyo-ku \\ Tokyo 113-0033 \\ Japan
}

\begin{abstract}
We study the identity projectors of the string field theory in the generic BCFT background. We explain how it can be identified as the projector in the linking algebra of the noncommutative geometry. We show that their (regularized) trace is exactly given by the boundary entropy which is proportional to the D-brane tension.
\end{abstract}

\footnotetext{
* E-mail address: matsuo@phys.s.u-tokyo.ac.jp
} 
In the previous letter [1], we argued that there are two types of the projectors for each of the Cardy state [2] in the cubic open string field theory [3] in the generic BCFT background. One is the sliver state discussed in many articles recently $4,5,6,6,8,8,9,10$ in the context of the vacuum string field theory. It is conjectured to describe the D-brane in the sense that it reproduces the D-brane tension in the level truncation [7] or the renormalization group flow [10] methods.

In this letter, however, we study the other projector which is the generalization of the identity operator. We are interested in this operator since it is interpreted as the nontrivial projector in the linking algebra [11, 12] which appear in the general framework of the noncommutative geometry [13, 14. In short, the linking algebra is a huge algebra of the open string fields with the star multiplication which link any pair of all D-branes which is possible from the bulk CFT. The detail will be explained in the text.

The identity operator also has a benefit that some exact calculations are possible for the topological invariants in the sense of the operator algebra K-theory [15, 16, 17, 18]. In particular the first nontrivial example, the trace of the projector, is exactly given as the boundary entropy [19] (see eq.(18)). This quantity was identified with the D-brane tension in [20]. It also appeared in the context of the boundary string field theory [21, 22]. It gives an explicit and analytic proof of the conjecture in [7] that the trace of the projector is proportional to the brane tension. In this sense, the identity projector is at least an equally legitimate candidate which represents the D-brane as the sliver projector.

As explained in the previous letter [1], our goal is to extend Witten's conjecture [23, 24] that the D-brane charge is classified by the K-theory to the operator theory of the conformal field theory. We hope that the result in this letter is a relevant step toward that direction.

We start from reviewing some of the essential features of the noncommutative soliton [25] in rather abstract language for the application to the string field theory algebra. In the noncommutative geometry, the commutative ring of the continuous functions over some topological space $X$ is replaced by the noncommutative $C^{*}$-algebra $\mathcal{A}$. The simplest example is the Moyal plane where $\mathcal{A}$ is given by the set of the bounded linear operators acting on the Hilbert space of the harmonic oscillators $\mathcal{B}(\mathcal{H})$. The noncommutative soliton is defined as the projector in this algebra,

$$
p \in \mathcal{A}, \quad p^{2}=p, \quad p^{*}=p .
$$


For the Moyal plane, the rank of the projector is interpreted as the soliton number,

$$
\tau(p)=\operatorname{Tr}_{\mathcal{H}} p=n \in \mathbf{Z}_{\geq \mathbf{0}}
$$

For the application to the D-brane [26], $\tau(p)$ is identified with the number of D-branes created after the tachyon condensation [27]. However, for the more general noncommutative space, the trace is not always quantized and may take a continuous spectrum. This is, actually, the major difference between the topological and the operator theoretical K-group. For example, in the noncommutative torus with the irrational noncommutative parameter $\theta$, the spectrum becomes dense between 0 and 1 with the following form [28, 12,

$$
\tau(p)=n+m \theta, \quad n, m \in \mathbf{Z},
$$

and it represents the composite system of $n$ D2 and $m$ D0 branes.

In the application to the string theory, this type of the projector is used to describe the lower dimensional D-brane charges which appear after the tachyon condensation [27] of the original D-brane described by the algebra $\mathcal{A}$. In the following, we argue that there are another type of the projectors which appears naturally in the open string field theory.

In general the open string may attach its two ends on the different Dbranes which have the different type of the noncommutative geometries. To describe such a situation, we need to consider the two D-branes (or the two $C^{*}$-algebras) as a whole. For that purpose, it is essential to introduce the notion of the Morita equivalence bimodule [13, 14, 29]. Two noncommutative geometries described by algebras $\mathcal{A}, \mathcal{B}$ is called Morita equivalent if there exists a bimodule ${ }^{\mathcal{A}} X^{\mathcal{B}}$ where $\mathcal{A}$ (resp. $\mathcal{B}$ ) acts from the left (right) on ${ }^{\mathcal{A}} X^{\mathcal{B}}$. In the string theory, this bimodule is identified with the open string that interpolates the different D-branes. In this bimodule we need to have $\mathcal{A}$ $\left(\mathcal{B}^{-}\right)$valued inner product $(,)_{\mathcal{A}}\left((,)_{\mathcal{B}}\right)$ on ${ }^{\mathcal{A}} X^{\mathcal{B}}$. They need to satisfy,

$$
\begin{aligned}
(a x, y)_{\mathcal{A}} & =a(x, y)_{\mathcal{A}}, \quad(x, \bar{a} y)_{\mathcal{A}}=(x, y)_{\mathcal{A}} a \\
(x, y b)_{\mathcal{B}} & =(x, y)_{\mathcal{B}} b, \quad(x \bar{b}, y)_{\mathcal{B}}=b(x, y)_{\mathcal{B}} \\
(x, y)_{\mathcal{A}} z & =x(y, z)_{\mathcal{B}}
\end{aligned}
$$

where $x, y, z \in{ }^{\mathcal{A}} X^{\mathcal{B}}$ and $a \in \mathcal{A}, b \in \mathcal{B}$. The string interpretation of these inner products is the star product of the string fields between the interpolating strings. We illustrate it in the Figure 1. The equations (4) are naturally interpreted as the associativity of the star product. We note that these abstract 

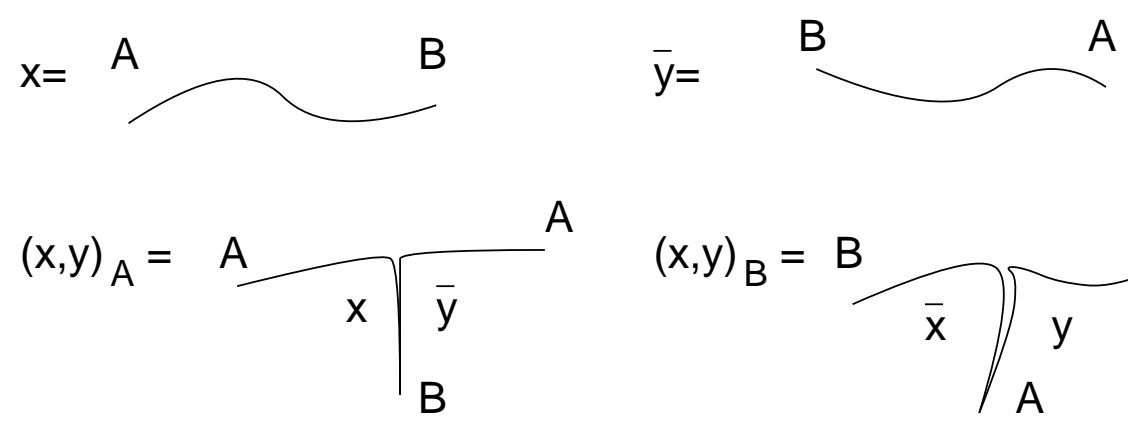

Figure 1: String interpretation of inner product of the bimodule

relations are also practically important to construct the noncommutative soliton [30, 12.

With the help of the Morita bimodule, one may define a new $C^{*}$-algebra $\mathcal{C}$ (which is called the linking algebra [11]),

$$
\mathcal{C}=\left\{\left(\begin{array}{cc}
a & x \\
\bar{y} & b
\end{array}\right) \mid a \in \mathcal{A}, b \in \mathcal{B}, x, y \in{ }^{\mathcal{A}} X^{\mathcal{B}}\right\} .
$$

The bar in $\bar{y}$ is the involution which turns the element of $\mathcal{A}-\mathcal{B}$ bimodule into the $\mathcal{B}-\mathcal{A}$ bimodule. This algebra can be interpreted as the open string system in the presence of two D-branes specified by $\mathcal{A}, \mathcal{B}$ [12]. In this composite system, we may define two obvious projectors as,

$$
p=\left(\begin{array}{cc}
1_{\mathcal{A}} & 0 \\
0 & 0
\end{array}\right), \quad q=\left(\begin{array}{cc}
0 & 0 \\
0 & 1_{\mathcal{B}}
\end{array}\right)
$$

which satisfies $p \mathcal{C} p=\mathcal{A}, q \mathcal{C} q=\mathcal{B}$ and $p+q=1_{\mathcal{C}}$. In this sense, $p$ and $q$ may be interpreted as the projectors to the algebras $\mathcal{A}$ and $\mathcal{B}$. They project into the single D-brane algebras from the composite system.

As mentioned before, these projectors are slightly different from those appearing in the noncommutative soliton (1). Firstly the projectors in (6) define the projections from the composite system of two D-branes but not from a single D-brane. Secondly, while the rank of the projector in (11) is usually finite, that of (6) may be infinite since it represents the rank of the whole algebra $\mathcal{A}($ or $\mathcal{B})$.

One may straightforwardly extend the notion of the linking algebra to the open string system with $N$ D-branes. In this case, the linking algebra $\mathcal{C}$ 
is described by $N$ by $N$ matrix and we have $N$ projectors which generalizes $p$ and $q$.

Let us proceed to apply this abstract idea to the more concrete setup of the BCFT. As we briefly reviewed in our previous letterf, the general strategy of the construction of the D-brane (the Cardy state) is as follows. Starting from the bulk CFT that defines the closed string, we have the algebraic data $\mathcal{I}$ which represents the set of the chiral primary fields $\phi_{i}(z)(i \in \mathcal{I})$. For each of the chiral primary fields, there exists a closed string state (the Ishibashi state [32]) |i $\rangle$. These states themselves, however, do not define the consistent boundary in the open string sector. For that purpose, we need to take their linear combination,

$$
\left.|a\rangle=\sum_{j} \frac{\psi_{a}^{j}}{\sqrt{S_{1 j}}}|j\rangle\right\rangle
$$

such that it reproduces the integer number of the chiral primary field in the open string channel as (after the modular transformation),

$$
\left\langle b\left|\tilde{q}^{\frac{1}{2}\left(L_{0}+\bar{L}_{0}-\frac{c}{12}\right)}\right| a\right\rangle=\sum_{i} \chi_{i}(q) n_{i a}{ }^{b},
$$

with $q=e^{2 \pi i \tau}$ and $\tilde{q}=e^{-2 \pi i / \tau}$. The constraint is that the coefficients,

$$
n_{i a}{ }^{b}=\sum_{j} \psi_{a}^{j}\left(\psi_{b}^{j}\right)^{*} \frac{S_{i j}}{S_{1 j}} .
$$

should be non-negative integers.

We suppose that the set of the Cardy state is labeled by $a \in \mathcal{V}$. In the rational conformal field theory, the set $\mathcal{V}$ is the finite set but in general we have the infinite number of the consistent boundary states. The boundary field ${ }^{a} \Psi_{i, \beta}^{b}(x)$ which represents the open string has four labels, $a, b \in \mathcal{V}$ for the two boundaries, $i \in \mathcal{I}$ for the chiral primary field and finally $\beta=1, \cdots, n_{i a}{ }^{b}$ for each channel. We write the highest weight state associated with this field as $\mid a, b ; i ; \beta>$.

In our previous letter [1], we proposed the string field theory algebra from these data. It is actually the linking algebra $\mathcal{C}$ defined as follows. Suppose we start from the D-brane system of $N_{a}$ D-branes of type $a$. The size of the linking algebra becomes $N \times N$ with $N=\sum_{a \in \mathcal{V}} N_{a}$. We label it by a pair of indices $\left(\left(a, I_{a}\right),\left(b, I_{b}\right)\right)$ with $I_{a}=1, \cdots, N_{a}$ or by $(A, B) A, B=1, \cdots, N$ if

\footnotetext{
${ }^{2}$ We use the notation of $[31]$ for the BCFT in this letter.
} 
we want to abbreviate it. The label $I_{a}$ are Chan-Paton index. The operators in $(A, B)$ th entry belongs to the set of the states of the form,

$$
\left\{L_{-n_{1}} \cdots L_{-n_{\ell}}|A, B ; i ; \beta>| i \in \mathcal{I}, \beta=1, \cdots, n_{i a}{ }^{b}\right\} .
$$

We denote the set of the Hilbert space as $\mathcal{H}_{A, B}=\mathcal{H}_{\left(a, I_{a}\right)\left(b, I_{b}\right)}$. The star product in the string field theory is defined as the mapping,

$$
\begin{aligned}
\star: & \mathcal{H}_{A B} \otimes \mathcal{H}_{B C} \rightarrow \mathcal{H}_{A C} \\
& |v>\otimes| w>\rightarrow \mid v \star w>=\operatorname{bpz}\left(\left\langle V_{3}|| v\right\rangle \otimes|w\rangle\right),
\end{aligned}
$$

where $\left\langle V_{3}\right|$ is the Witten's 3 string vertex operator and bpz is the BPZ conjugation. In [1] we argued that this algebra is in principle computed by the knowledge of the OPE of the boundary fields,

$$
{ }^{b} \Psi_{i, \alpha_{1}}^{c}\left(x_{1}\right)^{c} \Psi_{j, \alpha_{2}}^{a}\left(x_{2}\right)=\sum_{p, \beta, t}{ }^{(1)} F_{c p}\left[\begin{array}{cc}
i & j \\
b & a
\end{array}\right]_{\alpha_{1} \alpha_{2}}^{\beta t} \frac{1}{x_{12}^{\Delta_{i}+\Delta_{j}-\Delta_{p}}}{ }^{b} \Psi_{p, \beta}^{a}\left(x_{2}\right)+\cdots,
$$

and the conformal Ward identities derived in [⿴囗十 for the three string vertex. This linking algebra can be decomposed into the diagonal pieces $\mathcal{H}_{A A}$ which give the algebra on the D-brane $a$ and the off-diagonal pieces $\mathcal{H}_{A B}$ which define the Morita bimodule. As long as the label $A$ has of the form $\left(a, I_{a}\right)$ with the same $a$, the diagonal algebra $\mathcal{H}_{A A}$ are isomorphic with each other and we write it as $\mathcal{A}^{a}$.

One of the critical step here is to observe that the $C^{*}$-algebra $\mathcal{A}^{a}$ is unital for any Cardy state $|a\rangle$. This fact was implicitly written in [4] and explicitly stated in [1]. The identity operator $\mathcal{I}^{a}(a \in \mathcal{V})$ was defined through the identity chiral field in each $a$ - $a$ sector and the global conformal transformation 14

$$
w=\left(\frac{1+i z}{1-i z}\right)^{2}
$$

Explicitly an elegant expression was found in [33],

$$
\begin{aligned}
\mathcal{I}^{a} & =\left(\prod_{n=2}^{\infty} \exp \left\{-\frac{L_{-2^{n}}}{2^{n-1}}\right\}\right) e^{L_{-2}} \mid a, a ; 0> \\
& =\ldots e^{-\frac{1}{2^{2}} L_{-2^{3}}} e^{-\frac{1}{2} L_{-2^{2}}} e^{L_{-2}} \mid a, a ; 0>.
\end{aligned}
$$

From this operator, one may define the the analogue of $p$ and $q$ in (6). We use the similar symbol $\mathcal{I}^{A}$ as the projector where the identity operator $\mathcal{I}^{a}$ is 
located at $A A$-th entry $\left(A\right.$ is of the form $\left.\left(a, I_{a}\right)\right)$ and zero elsewhere. They satisfy

$$
\sum_{A=1}^{N} \mathcal{I}^{A}=1_{\mathcal{C}}, \quad \mathcal{I}^{A} \star \mathcal{I}^{B}=\delta_{A B} \mathcal{I}^{B}, \quad \mathcal{I}^{A} \star \mathcal{C} \star \mathcal{I}^{A} \sim \mathcal{A}^{a}
$$

or

$$
\mathcal{I}^{A} \star\left|B, C>=\delta_{A B}\right| B, C>, \quad\left|B, C>\star \mathcal{I}^{A}=\delta_{C A}\right| B, C>
$$

for any $\mid B, C>\in \mathcal{H}_{B C}$ embedded into the $B C$ th entry. As we discuss in the definition of the linking algebra, we would like to interpret this projector as characterizing each D-brane.

In the following, we would like to calculate the trace of $\mathcal{I}^{a}$. Usually it has been argued that trace diverges and such kind of the calculation is meaningless. There is, however, a systematic method to regularize the infinity and we conjecture that the finite outcome will have the meaningful interpretation.

Since $\mathcal{I}^{a}$ is the identity element in the algebra $\mathcal{A}^{a}$, we first evaluate the number of the generators of $\mathcal{A}^{a}$ which is identified with the number of states $\left|\mathcal{H}_{a a}\right|$. (For the simplicity we omit Chan-Paton indices and replace the index $A, B, \cdots$ by Cardy state index $a, b, \cdots$ ) As in the calculation of the character, we regularize the counting of the states by introducing $\tilde{q}^{L_{0}-c / 24}$ with $\tilde{q}=$ $e^{-2 \pi i / \tau}$ and take the $\tilde{q} \rightarrow 1(\tau \rightarrow i \infty)$ limit later.

$$
\begin{aligned}
\left|\mathcal{H}_{a a}\right| & =\lim _{\tilde{q} \rightarrow 1} \operatorname{Tr}_{\mathcal{H}_{a a}} \tilde{q}^{L_{0}-c / 24}=\lim _{\tilde{q} \rightarrow 1} \sum_{i} n_{i a}{ }^{a} \chi_{i}(\tilde{q}) \\
& =\lim _{q \rightarrow 0}\left\langle a\left|q^{\frac{1}{2}\left(L_{0}+\bar{L}_{0}\right)}\right| a\right\rangle \\
& =|\langle\operatorname{vac} \mid a\rangle|^{2}
\end{aligned}
$$

with $q=e^{2 \pi i \tau}$. Here $\chi_{i}(q)$ is the character for the chiral primary field $\phi_{i}$ and $\langle\mathrm{vac}|$ is the closed string vacuum state. In passing from the first to the second line, we used eq.(8). The final answer becomes finite! Indeed this type of the calculation is typical in the BCFT, for example, in the norm calculation of the Ishibashi state (see for example [31]).

We have to be careful not to confuse this quantity as the trace of $\mathcal{I}^{a}$. In the finite $N \times N$ matrix case, the number of the generators of the algebra is $N^{2}$ but the trace of the identity operator is $N$. While it may be naive to apply it to the infinite $N$ case, we have to use this analogy to conclude the trace formula for $\mathcal{I}^{a}$,

$$
\tau\left(\mathcal{I}^{a}\right)=\langle\operatorname{vac} \mid a\rangle
$$


As we mentioned at the beginning of this letter, this quantity is called the boundary entropy [19] and gives the tension of the D-brane [20, 21, 22]. We think that this result is quite satisfactory and one may safely conjecture that the identity projector is a candidate to represent the D-brane in the open string field theory.

More generally the projection $p$ to the composite system of $n_{a}$ D-branes of the type $a$ has the trace,

$$
\tau(p)=\sum_{a \in \mathcal{V}} n_{a}\langle\operatorname{vac} \mid a\rangle
$$

which may be regarded as the generalization of the formula for the quantum torus (3). The integrability of the D-branes appear in the coefficients but $\tau(p)$ as a whole is not necessarily the multiple of the integer.

To conclude this letter, we would like to give some comments.

1. We summarize the reason why we believe that the rank infinite projector is more natural than the rank finite ones. Suppose we have a projector $p$ which describes a D-brane system. It gives a subalgebra of the original open string field theory algebra $\mathcal{C}$ in the form $\mathcal{A}=p \mathcal{C} p$. If $p$ is the rank finite projector, $\mathcal{A}$ is isomorphic to the finite size matrix algebra and should be identified as the group of the Chan-Paton factor. On the other hand, if we use our infinite rank projector, $\mathcal{A}$ is the subalgebra of $\mathcal{C}$ generated by infinite number of the open strings whose two ends are attached to the given D-brane we would like to pick up. We think that the rank finite algebra is too simple to be identified as the algebra generated by the open string fields.

2. We have pointed out that there are some differences between the conventional noncommutative soliton and the projectors in the linking algebra. This difference, however, should be superficial. If the D-brane in the higher dimension can be decomposed into the collection of the lower dimensional D-branes, it simply implies that the Cardy state associated with the higher dimensional brane is reducible and can be decomposed into the sum of the irreducible ones,

$$
|a\rangle=\left|a_{1}\right\rangle+\left|a_{2}\right\rangle+\cdots .
$$

In such a situation, the $C^{*}$-algebra of the higher dimensional D-brane is actually considered as the linking algebra of the lower dimensional Dbranes and the projectors of the both approaches become the identical. 
It is of some interest to check that such a decomposition is possible in the tachyon condensation of the noncommutative D-branes [26]. A plausible answer is that the algebra of the open string fields of $D(p+2)$ brane can be factorized as $\mathcal{C}=\mathcal{B}(\mathcal{H}) \otimes \mathcal{A}$ where $\mathcal{A}$ is the algebra of $D$ $p$-brane. If this guess is true, the rank finite projector $p$ in [26] should be mapped to the rank infinite projector of the string fields as $p \otimes 1_{\mathcal{A}}$.

3. From the viewpoint of the noncommutative geometry, the quantity (18) is the simplest geometrical invariant made from the projector. The general invariants are constructed by pairing with the cyclic cohomology element $\tau_{n}$ [15]

$$
\tau_{n}\left(\mathcal{I}^{a}, \cdots, \mathcal{I}^{a}\right) .
$$

We would like to know how the notion of the cyclic cocycle can be extended to the string field theory algebra. In the case of the commutative situation, such invariants are expressed by using the differentiation of the projector. In the operator language, we need to use the operator product expansion (12) to express such operation. It seems to us an essential step to seek such a possibility to explore the K-group of the string field theory algebra and the D-brane charge [23].

Acknowledgement: The author would like thank T. Eguchi, T. Kawano, T. Takayanagi and K. Ohmori for the useful conversations.

The author is supported in part by Grant-in-Aid (\#13640267) and in part by Grant-in-Aid for Scientific Research in a Priority Area "Supersymmetry and Unified Theory of Elementary Particle" (\#707) from the Ministry of Education, Science, Sports and Culture.

\section{References}

[1] Y. Matsuo, "BCFT and Sliver state", hep-th/0105175.

[2] J. L. Cardy, Nucl. Phys. B324 (1989) 581-596.

[3] E. Witten, Nucl. Phys. B268 (1986) 253.

[4] L. Rastelli and B. Zwiebach, "Tachyon potentials, star products and universality", hep-th/0006240. 
[5] V. A. Kostelecký and R. Potting, Phys. Rev. D63 (2001) 046007, hep-th/0008252.

[6] L. Rastelli, A. Sen and B. Zwiebach, "String Field Theory Around The Tachyon Vacuum", hep-th/001225.

[7] L. Rastelli, A. Sen and B. Zwiebach, "Classical Solutions in String Field Theory Around the Tachyon Vacuum", hep-th/0102112.

[8] L. Rastelli, A. Sen and B. Zwiebach, "Half-strings, Projectors and Multiple D-branes in Vacuum String Field Theory", hep-th/0105058.

[9] D. J. Gross, W. Taylor, "Split string field theory I", hep-th/0105059.

[10] L. Rastelli, A. Sen, B. Zwiebach, "Boundary CFT Construction of Dbrane in Vacuum String Field Theory", hep-th/0105168.

[11] For example, I. Raeburn and D. P. Williams, "Morita Equivalence and Continuous-Trace $C^{*}$-algebra", (AMS 1991) section 3.2.

[12] H. Kajiura, Y. Matsuo, T. Takayanagi, "Exact Tachyon Condensation On Noncommutative Torus", hep-th/0104143.

[13] A. Connes, M. Douglas and A. Schwarz, JHEP 9802 (1998) 003 , hep-th/9711162.

[14] N. Seiberg and E. Witten, JHEP 9909 (1999) 032, hep-th/9908142.

[15] A. Connes, "Noncommutative geometry" (Academic Press, 1994).

[16] N. E. Wegge-Olsen, "K-theory and $C^{*}$-algebras", (Oxford, 1993).

[17] Y. Matsuo, Phys. Lett. B 499 (2001) 223 , hep-th/0009002.

[18] J. A. Harvey and G. Moore, "Noncommutative tachyons and K-theory," hep-th/0009030.

[19] I. Affleck and A. W. Ludwig, Phys. Rev. Lett. 67 (1991) 161;

I. Affleck and A. W. Ludwig, Phys. Rev. B48 (1993) 7297.

[20] J. Harvey, S. Kachru, G. Moore and E. Silverstein, JHEP 0003 (2000) 001, hep-th/9909072. 
[21] D. Kutasov, M. Marino, G. Moore, JHEP 0010 (2000) 045, hep-th/0009148.

[22] S.P. de Alwis, Phys. Lett. B505 (2001) 215-221, hep-th/0101200.

[23] E. Witten, JHEP9812 (1998) 019, hep-th/9810188.

[24] E. Witten, Int. J. Mod. Phys. A16 (2001) 693-706, hep-th/0007175.

[25] P. Gopakumar, S. Minwalla, A. Strominger, JHEP 0006 (2000) 022, hep-th/0003160.

[26] J. A. Harvey, P. Kraus, F. Larsen and E. J. Martinec, JHEP0007 (2000) 042, hep-th/0005031.

[27] A. Sen, "Non-BPS states and branes in string theory", hep-th/9904207 and references therein.

[28] I. Bars, H. Kajiura, Y. Matsuo, T. Takayanagi, Phys. Rev. D63 (2001) 086001, hep-th/0010101.

[29] A. Schwarz, "Morita equivalence and duality", hep-th/9805034.

[30] E. Martinec and G. Moore, "Noncommutative soliton on orbifolds", hep-th/0101199.

[31] R. E. Behrend, P. A. Pearce, V. B. Petkova, J-B. Zuber, Nucl. Phys. B570 (2000) 525-589, Nucl. Phys. B579 (2000) 707-773, hep-th/9908036.

[32] N. Ishibashi, Mod. Phys. Lett. A4 (1987) 251.

[33] I. Ellwood, B. Feng, Y. He and N. Moeller, "The identity string field and the tachyon vacuum", hep-th/0105024. 